\title{
Theory of the $c$-Axis Penetration Depth in the Cuprates
}

\author{
R. J. Radtke and V. N. Kostur甘 \\ Center for Superconductivity Research, Department of Physics, \\ University of Maryland, College Park, Maryland, 20742-4111 \\ K. Levin \\ Department of Physics and The James Franck Institute, \\ The University of Chicago, Chicago, Illinois 60637
}

\begin{abstract}
Recent measurements of the London penetration depth tensor in the cuprates find a weak temperature dependence along the $c$-direction which is seemingly inconsistent with evidence for $d$-wave pairing deduced from in-plane measurements. We demonstrate in this paper that these disparate results are not in contradiction, but can be explained within a theory based on incoherent quasiparticle hopping between the $\mathrm{CuO}_{2}$ layers. By relating the calculated temperature dependence of the penetration depth $\lambda_{c}(T)$ to the $c$-axis resistivity, we show how the measured ratio $\lambda_{c}^{2}(0) / \lambda_{c}^{2}(T)$ can provide insight into the behavior of $c$-axis transport below $T_{c}$ and the related issue of "confinement."
\end{abstract}

PACS numbers: 74.20.-z. 74.25.-q, 74.25.Nf, 74.25.Fy

Measurements of the temperature dependence of the in-plane penetration depth in $\mathrm{YBa}_{2} \mathrm{Cu}_{3} \mathrm{O}_{7-\delta}$ (YBCO) have been interpreted asproviding strong support for a $d$-wave order parameter 1 With the availability of highquality single crystals, these measurements arenow being extended to the $c$-axis direction in $\mathrm{YBCO}^{2} \mathrm{~B}$ as well as in $\mathrm{Bi}_{2} \mathrm{Sr}_{2} \mathrm{CaCu}_{2} \mathrm{O}_{8}$ (BSCCO) 1 and $\mathrm{La}_{1-\mathrm{x}} \mathrm{Sr}_{\mathrm{x}} \mathrm{CuO}_{4}$ (LSCO). 0 There appears to be one consistent feature of these different experiments: the (low) temperature dependence of the $c$-axis penetration depth $\lambda_{c}(T)$ is much weaker than that observed in the $a b$ plane. In addition, the penetration depth ratio $\lambda_{c}^{2}(0) / \lambda_{c}^{2}(T)$ in YBCO is linear in $T$ at low $T$ with a small slope that decreases with decreasing oxygen content.2

The present paper addresses these penetration depth data in conjunction with the $c$-axis resistivity $\rho_{c}(T)$ for a variety of different cuprates. We argue that the temperature dependence of $\rho_{c}(T)$ essentially determines that of $\lambda_{c}(T)$; in particular, $\lambda_{c}(T)$ is expected to have a weak temperature dependence at low $\mathrm{T}$ whenever $\rho_{c}(T)$ exhibits a strong semiconducting behavior. In this way, we can reconcile the $a b$-plane data supporting $d$-wave pairing with the observed $c$-axis behavior. In addition, by presenting typical results for $\lambda_{c}$ with the corresponding $\rho_{c}$, we illustrate the generic features of these quantities without relying on specific parameterizations of (or fits to) existing data. Through the study of the $c$-axis coupling, we touch on the issues which may lie at the heart of the nature of the normal state ${ }^{6}$ and also potentially the mechanism of high-temperature superconductivity. In particular, our results imply that a Fermi-liquid-based description of the electronic states in the $\mathrm{CuO}_{2}$ planes appears as consistent with the data as theories based on the idea of "confinement," even though the underlying assumptions are considerably different.

To understand the behavior of $\lambda_{c}(T)$ and the nature of $c$-axis coupling below $T_{c}$, we utilize an incoherent hopping model for the $c$-axis coupling 111 Above the critical temperature $T_{c}$, this model views each $\mathrm{CuO}_{2}$ layer as a two- dimensional Fermi liquid which is weakly coupled to its nearest neighbors. This model yields a semiconducting $T$ dependence of $\rho_{c}(T)\left(d \rho_{c}(T) / d T<0\right)$ while maintaining a finite residual resistivity $\rho_{c}(0)$. The semiconducting behavior is also associated with the absence of a Drude peak in the $c$-axis optical conductivity 6 Thus, while the $c$-axis transport properties suggest an insulating state at low temperatures, the actual zero-temperature state is nevertheless metallic. comparing its predictions with the available experimental data provides an opportunity to learn about the precise low- $T$ behavior of the $c$-axis resistivity as well as the degree to which the layers communicate in the superconducting phase.

We begin by writing the Hamiltonian for the electronic system as the sum of a Hamiltonian for the individual $\mathrm{CuO}_{2}$ layers, $\sum_{m} H_{m}$, and an interlayer coupling term $H_{\perp}: H_{\mathrm{el}}=\sum_{m} H_{m}+H_{\perp}$. We leave the intra-layer Hamiltonian unspecified except to demand that each $H_{m}$ yield a two-dimensional Fermi liquid which becomes a either an $s$ - or a $d$-wave superconductor below a critical temperature $T_{c} . H_{\perp}$ is taken to be 41

$$
H_{\perp}=\sum_{i m \sigma} t_{i m} c_{i, m+1, \sigma}^{\dagger} c_{i m \sigma}+\text { h.c. }
$$

where $t_{i m}=t_{\perp}+V_{i m}+\sum_{j} g_{i-j, m} \phi_{j m}$ and $c_{i m \sigma}$ is the usual quasiparticle annihilation operator for site $i$ in layer $m$ and spin projection $\sigma$. Physically, the interlayer coupling arises from quasiparticle hopping due to wavefunction overlap (parameterized by $t_{\perp}$ ), impurity scattering (modeled by the random variable $V_{i m}$ ), and bosonic scattering (represented by the field $\phi_{i m}$ which couples to the electronic quasiparticles with strength $g_{i m}$ ).

In treating $H_{\perp}$, we are guided by two observations: (1) the mean free path in the $c$-direction extracted from normal-state transport measurements is less than the lattice spacing, 1213 and (2) the $c$-axis properties in the superconducting state are consistent with a picture 
where nearest=neighbor $\mathrm{CuO}_{2}$ layers form an SIS tunnel junction. 13.14 These observations suggest that the $c$-axis transport may be viewed as an incoherent tunnelipg precess. Several theories of this incoherence exist, 08 , 12 , 15 but for simplicity we adopt the phenomenological model of Ref. 8 and simulate the effects of incoherence by performing our calculations to second order in $H_{\perp}$. This procedure immediately yields an intrinsic Josephson effect 10 and a reasonable magnitude and $T$ dependence of the $c$-axis resistivity 8010 . 6

In the normal state, we can calculate the $c$-axis dc conductivity by direct analogy with the problem of tunpeling in a normal metal-insulator-normal metal junction 6 . 1 . . 7 .7 and obtain a $c$-axis conductivity which is the sum of the conductivities due to each process: $\sigma_{c}=\sigma_{c}^{\text {direct }}+\sigma_{c}^{\text {imp }}+$ $\sigma_{c}^{\text {inel } 10}$ Generalizing the results of Ref. 10 to anisotropic scattering, we can write these terms as

$$
\begin{gathered}
\sigma_{c}^{\text {direct }}=\sigma_{0} N_{0} t_{\perp}^{2}\left(\frac{\tau_{a b}}{\pi \hbar}\right), \\
\sigma_{c}^{\text {imp }}=\sigma_{0} N_{0}^{2}\left\langle\left\langle\left|V_{\mathbf{k}-\mathbf{k}^{\prime}}\right|^{2}\right\rangle_{\mathbf{k}}\right\rangle_{\mathbf{k}^{\prime}},
\end{gathered}
$$

and

$$
\sigma_{c}^{\text {inel }}=\sigma_{0} N_{0}^{2}\left\langle\left\langle\left|g_{\mathbf{k}-\mathbf{k}^{\prime}}\right|^{2}\right\rangle_{\mathbf{k}}\right\rangle_{\mathbf{k}^{\prime}} \frac{\hbar \Omega_{0} / 2 k_{B} T}{\sinh ^{2}\left(\hbar \Omega_{0} / 2 k_{B} T\right)} .
$$

Here, $N_{0}$ is the density of states per unit cell per spin at the Fermi surface, $\hbar / \tau_{a b}$ is the intra-layer scattering rate, $\sigma_{0}=\left(4 \pi e^{2} / \hbar\right)\left(d / a^{2}\right), d(a)$ is the inter- (intra-) layer unit cell dimension, $e$ is the electronic charge, and the angle brackets denote a normalized average over the Fermi surface. For simplicity, we take the interlayer inelastic scattering to have an Einstein spectrum with frequency $\Omega_{0}$ but keep the wave-vector dependence in the impurity$\left(V_{\mathbf{q}}\right)$ and boson-assisted $\left(g_{\mathbf{q}}\right)$ hopping amplitudes.

The calculation of the penetration depth in the superconducting state may be performed either by noting that the $c$-axis critical current $\propto 1 / \lambda_{c}^{2}$ in layered superconductors 18 and computing the critical current in the standard way, 19,10 or by calculating the optical conductivity and extracting the penetration depth from the imaginary part.11 In either case, one obtains

$$
\frac{1}{\lambda_{c}^{2}}=\frac{32 \pi e^{2} d}{\hbar^{2} c^{2} a^{2}} \frac{T^{2}}{N_{\|}^{2}} \sum_{k_{l} k_{l^{\prime}}^{\prime}} t^{2}\left(k_{l}, k_{l^{\prime}}^{\prime}\right) F\left(k_{l}\right) F\left(k_{l^{\prime}}^{\prime}\right),
$$

where $c$ is the speed of light, $T$ is the temperature $\left(k_{B}\right.$ $=1), N_{\|}$is the number of lattice sites in a single layer, $k_{l}=\left(\mathbf{k}, i \omega_{l}\right), F\left(k_{l}\right)$ is the Gor'kov propagator, 20121 and $t^{2}\left(k_{l}, k_{l^{\prime}}^{\prime}\right)$ is the generalized interlayer hopping matrix element. As with the conductivity, this matrix element is the sum of the matrix elements due to direct scattering, $t^{2 \text {,direct }}\left(k_{l}, k_{l^{\prime}}^{\prime}\right)=\frac{N_{\|}}{T} \delta_{\mathbf{k}, \mathbf{k}^{\prime}} \delta_{l, l^{\prime}} t_{\perp}^{2}$; impurity-assisted scattering, $t^{2, \operatorname{imp}}\left(k_{l}, k_{l^{\prime}}^{\prime}\right)=\frac{1}{T} \delta_{l, l^{\prime}}\left|V_{\mathbf{k}-\mathbf{k}^{\prime}}\right|^{2}$; and bosonassisted scattering, $t^{2, \text { inel }}\left(k_{l}, k_{l^{\prime}}^{\prime}\right)=\left|g_{\mathbf{k}-\mathbf{k}^{\prime}}\right|^{2} D\left(k_{l}-k_{l^{\prime}}^{\prime}\right)$.
In the last term, $D\left(k_{l}\right)$ is the propagator for the interlayer boson. We note that all three mechanisms produce a contribution to the penetration depth. This result is not surprising, given the close similarity between incoherently coupled layers and tunnel junctions: the direct and impurity-assisted hopping processes formally resemble the processes considered in conventional SIS junctions $222,24,29$ and boson-assisted hopping has been known to contribute to tunneling in superconducting junctions in both the quasiparticle 25 and Josephson 24 channels for some time.

To compute the penetration depth from these formulae, we make several simplifying assumptions. First, we use the standard BCS form for the Gor'kov propagators and perform the sums over $\mathbf{k}$ in the usual way by restricting the wave vectors of the self-energies and matrix elements to the Fermi surface and then integrating the remaining energy dependence from $-\infty$ to $+\infty$. We reiterate that the weak interlayer coupling allows us to perform our calculations to second order in the interlayer hopping amplitudes, and this implies that inter-layer scattering effects which act toduce $1 / \lambda_{c}^{2}$ are of higher order and can be neglected 10 Second, we make the reasonable approximation that the layers are identical. Third, we account for the anisotropy of the hopping processes by taking the impurity scattering matrix element $\left|V_{\mathbf{k}-\mathbf{k}^{\prime}}\right|^{2}$ to have a Lorentzian form with a maximum at $\mathbf{k}=\mathbf{k}^{\prime}$ and a half width $\delta k / k_{F}=0.01$ and by assuming that the wave-vector structure of $\left|g_{\mathbf{k}-\mathbf{k}^{\prime}}\right|^{2}$ is such that the bosonassisted processes contribute to both the resistivity and the penetration depth with the same strength. The qualitative trends in $\rho_{c}$ and $\lambda_{c}$ we discuss do not depend on these choices; see Ref. 11 for details.

From these relations, we see that $\rho_{c}=1 / \sigma_{c}$ is determined by the same parameters as $\lambda_{c}$. We are therefore able to connect the two quantities and examine the qualitative predictions of our theory. Fig. 11 shows the resulting curves for $\rho_{c}(T)$ and the corresponding $c$-axis penetration depth ratio for both $s$ - and $d$-wave pairing in several limiting cases of our model.

If only direct hopping is present [cf. Fig. 11(a)], only those terms corresponding to wave function overlap contribute to the resistivity and the penetration depth. This case may be relevant for materials like fully oxygenated YBCO, which is one of the least anisotropic cuprates $\left(\rho_{c}\left(T_{c}\right) / \rho_{a b}\left(T_{c}\right) \sim 10-10^{2}\right.$ compared to $\rho_{c}\left(T_{c}\right) / \rho_{a b}\left(T_{c}\right) \sim$ $10^{5}$ for BSCCO ${ }^{13}$ ) and therefore potentially the least incoherent. In this instance, $\rho_{c}$ reflects the temperature dependence, though not the magnitude, of the $a b$-plane resistivity: $\rho_{c} \propto \rho_{a b} \propto T$ [cf. Eq. (2)]. The resulting penetration depth is also marked by this near-coherence and has the same temperature dependence for both the $a b$ - and $c$-axis directions, regardless of pairing symmetry.

The case of assisted hopping, where only the impurityand boson-assisted processes contribute to the $c$-axis transport, is shown in Fig. 1(b). Materials like BSCCO with $c$-axis mean free paths much less than the lattice 
spacing are expected to be close to this limit. $\rho_{c}$ in this case is marked by a negative temperature derivative which gives rise to a semiconducting temperature dependence above $T_{c}$. This upturn in the resistivity is due to the freezing-out of the inelastic interlayer scattering at low $T$, which inhibits $c$-axis transport and therefore increases the resistivity. At lower $T$, however, the impurity scattering acts to limit the conductivity, and $\rho_{c}(T)$ saturates. The $c$-axis penetration depth is also modified by the incoherent transport and becomes distinct from the $a b$-plane result. For both pairing symmetries, the $c$-axis penetration depth ratio is larger than the $a b$ plane penetration depth ratio at all temperatures. In particular, the $d$-wave penetration depth ratio $\lambda_{c}^{2}(0) / \lambda_{c}^{2}(T)$ resembles the $s$-wave case, although the low-temperature behavior is still a power law. In addition, the difference between in-plane and $c$-axis penetration depth ratios is much more pronounced for $d$-wave pairing than for $s$ wave paring (see the upper inset in Fig. 1 (b)). Moreover, the temperature dependence in the $d$-wave case is close to, and may be experimentally indistinguishable from, the ( $s$-wave) Ambegaokar-Baratoff23 form.

In the intermediate case, all processes contribute to $c$-axis transport [Fig. 1ㅡ(c)]. Compounds such as deoxygenated YBCO may belong to this class. As in the assisted hopping case, $\rho_{c}$ looks semiconducting at high temperatures. At low temperatures, however, the inplane scattering rate is reduced, and this leads to a low- $T$ conductivity dominated by direct hopping processes [cf. Eq. (2)]. The net result is a peak in $\rho_{c}$ which may lie below $T_{c}$. In contrast to the resistivity, the penetration depth ratio shows no new behavior in this case, but is mid-way between the direct result, where both $a b$ - and $c$-axis penetration depth ratios are the same, and the assisted hopping result, where they are considerably different. Note especially that the low-temperature $c$-axis penetration depth ratio for $d$-wave pairing is clearly linear in $T$ but with a much smaller slope than its in-plane counterpart.

Fundamentally, the behavior discussed above results from the different characteristic temperature dependences that arise when the interlayer coupling is very weak (incoherent) as opposed to when it is strong (coherent). In a material with very weak interlayer coupling, quasiparticle transport between adjacent layers is analogous to tunneling in NIN or SIS junctions. This tunneling is mediated by both elastic are inelastic scattering and is therefore associated with a semiconducting $c$-axis resistivity in the normal state and a very low slope of the $c$-axis penetration depth ratio at low temperatures. The small low-temperature slope of $\lambda_{c}^{-2}(T)$ is a special feature of specular Josephson tunneling and occurs in either $s-23$ or $d$-wave 11 superconductors. On the other hand, in materials with stronger interlayer coupling, quasiparticle transport is nearly coherent and so quasi-three-dimensional results obtain: $\rho_{c}$ is metallic, and $\lambda_{c}^{2}(0) / \lambda_{c}^{2}(T)$ possesses a larger low- $T$ slope for $d$ wave pairing relative to the weakly coupled case.
Empirically, therefore, one should associate a reduction in the low- $T$ slope of the $c$-axis penetration depth ratio in either $s$ - or $d$-wave superconductors with a decrease in the interlayer coupling and a semiconducting temperature dependence of $\rho_{c}$. Our theory thus accommodates the currently available data in both the intraand interlayer directions despite their very different lowtemperature slopes. Moreover, this consistency suggests that the cuprates may indeed be $d$-wave superconductors. While our theory can explain the qualitative features of the present experimental data, these data are incomplete; further systematic experiments on different cuprates and for particular cuprates with different stoichiometries are clearly called for to further test the trends reported in this paper. In addition, further theoretical effort is required to understand the relationship of our theory to others in the literature and to provide a way of distinguishing them experimentally.

Among these other theories of $c$-axis coupling, the "confinement" approach 6 is worth discussing further, since we come to similar conclusions despite vastly different starting assumptions. In contrast to our Fermi-liquidbased approach, the confinement theory asserts that each $\mathrm{CuO}_{2}$ layer is a spin-charge separated tomographic Luttinger liquid. 6 Nevertheless, the expressions for the interlayer transport which arise in this model are similar to those in this work with two important differences: (1) the Green's functions used in the calculation correspond to a Luttinger and not a Fermi liquid, and (2) inelastic scattering is an intrinsic part of the interlayer transport process and is not extrinsic to the layers as in, for example, the boson-assisted hopping term in our theory. Despite these differences, both theories obtain a semiconducting $T$ dependence of $\rho_{c} 6$ and a small low- $T$ slope of the penetration depth ratio, 26 in agreement with experiments. This comparison suggests that models of $c$-axis coupling in the cuprates should view interlayer transport as a tunneling process with a strong inelastic component, but the detailed origin of this effect may not be readily extracted from the currently available experiments.

The authors would like to thank D. A. Bonn and S. Kamal for making their penetration depth data available to us, and T. Timusk and S. Anlage for valuable discussions. This work was supported by the National Science Foundation (DMR-9123577 and DMR 91-20000, through the Science and Technology Center for Superconductivity) and by NASA Grant No. NAG3-1395.

* Address after September 1, 1995: Department of Physics, SUNY at Stony Brook, Stony Brook, New York 117943800 .

${ }^{1}$ W. N. Hardy et al., Phys. Rev. Lett. 70, 3999 (1993).

${ }^{2}$ D. A. Bonn et al., J. Phys. Chem. Solids (to appear); C. 
C. Homes et al., submitted to Physica C.

${ }^{3}$ Note, however, that there is currently no consensus on the precise shape of $\lambda_{c}^{2}(0) / \lambda_{c}^{2}(T)$ in YBCO: some measurements reveal an SNS-like $T$ dependence [cf. J. Mao et al., Phys. Rev. B 51, 3316 (1995)], while others see a more conventional form. 2 Since our theory cannot account for the former, we focus on the latter and await further experimental clarification of the situation.

${ }^{4}$ R. Kleiner, F. Steinmeyer, G. Kunkel, and P. Müller, Phys. Rev. Lett. 68, 2394 (1992); R. Kleiner and P. Müller, Phys. Rev. B 49, 1327 (1994).

${ }^{5}$ T. Shibauchi et al., Phys. Rev. Lett. 72, 2263 (1994).

${ }^{6}$ P. W. Anderson and Z. Zou, Phys. Rev. Lett. 60, 132 (1988); ibid. 60, 2557 (1988); and D. G. Clarke, S. P. Strong, and P. W. Anderson, Phys. Rev. Lett. 74, 4499 (1995).

7 S. Chakravarty, A. Sudbø, P. W. Anderson, and S. Strong, Science 261, 337 (1993); A. Sudbø, S. Chakravarty, S. Strong, and P. W. Anderson, Phys. Rev. B 49, 12245 (1994).

${ }^{8}$ A. G. Rojo and K. Levin, Phys. Rev. B 48, 16861 (1993).

${ }^{9}$ R. J. Radtke, C. N. Lau, and K. Levin, J. Supercon., August, 1995 (to appear).

${ }^{10}$ R. J. Radtke and K. Levin, Physica C 250 (to appear).

${ }^{11}$ R. J. Radtke and V. N. Kostur, to be published.

${ }^{12}$ A. J. Leggett, Braz. J. Phys. 22, 129 (1992).

${ }^{13}$ For a review, see S. L. Cooper and K. E. Gray, in Physical Properties of High-Temperature Superconductors IV, D. M. Ginsberg, ed. (World Scientific, Singapore, 1994).

14 J. D. Hettinger et al., Phys. Rev. Lett. 74, 4726 (1995).

${ }^{15}$ M. J. Graf, D. Rainer, and J. A. Sauls, Phys. Rev. B 47, 12089 (1993), and unpublished; M. J. Graf, M. Palumbo, D. Rainer, and J. A. Sauls, unpublished.

${ }^{16}$ See Refs. 10 and 11 for a detailed discussion.

${ }^{17}$ P. B. Littlewood and C. Varma, Phys. Rev. B 45, 12636 (1992).

18 L. N. Bulaevskii, Sov. Phys.-JETP 37, 1133 (1973); J. R. Clem, Physica (Amsterdam) 162-164C, 1137 (1989).

${ }^{19}$ G. D. Mahan, Many-Particle Physics (Plenum, New York, 1986), Ch. 9.

${ }^{20}$ A. A. Abrikosov, L. P. Gor'kov, and I. I. Dzyaloshinski, Methods of Quantum Field Theory in Statistical Physics (Dover, 1962).

${ }^{21}$ P. B. Allen and B. Mitrović, in Solid State Physics, Vol. 37, H. Ehrenreich, F. Seitz, and D. Turnbull, eds. (Academic Press, New York, 1982), p. 1.

${ }^{22}$ M. H. Cohen, L. M. Falicov, and J. C. Phillips, Phys. Rev. Lett. 8, 316 (1962).

${ }^{23}$ V. Ambegaokar and A. Baratoff, Phys. Rev. Lett. 10, 486 (1963); ibid. 11, 104 (1963).

${ }^{24}$ C. B. Duke, Tunneling in Solids (Academic Press, New York, 1969).

${ }^{25}$ L. Kleinman, B. N. Taylor, and E. Burstein, Rev. Mod. Phys. 36, 208 (1964); J. R. Tucker and M. J. Feldman, Rev. Mod. Phys. 57, 1055 (1985).

${ }^{26}$ While preparing this manuscript, we received a preprint by T. Xiang and J. M. Wheatley which computes the anisotropic penetration depth within the pair hopping model associated with the confinement picture that obtains results for $\lambda_{c}^{2}(0) / \lambda_{c}^{2}(T)$ similar to ours.
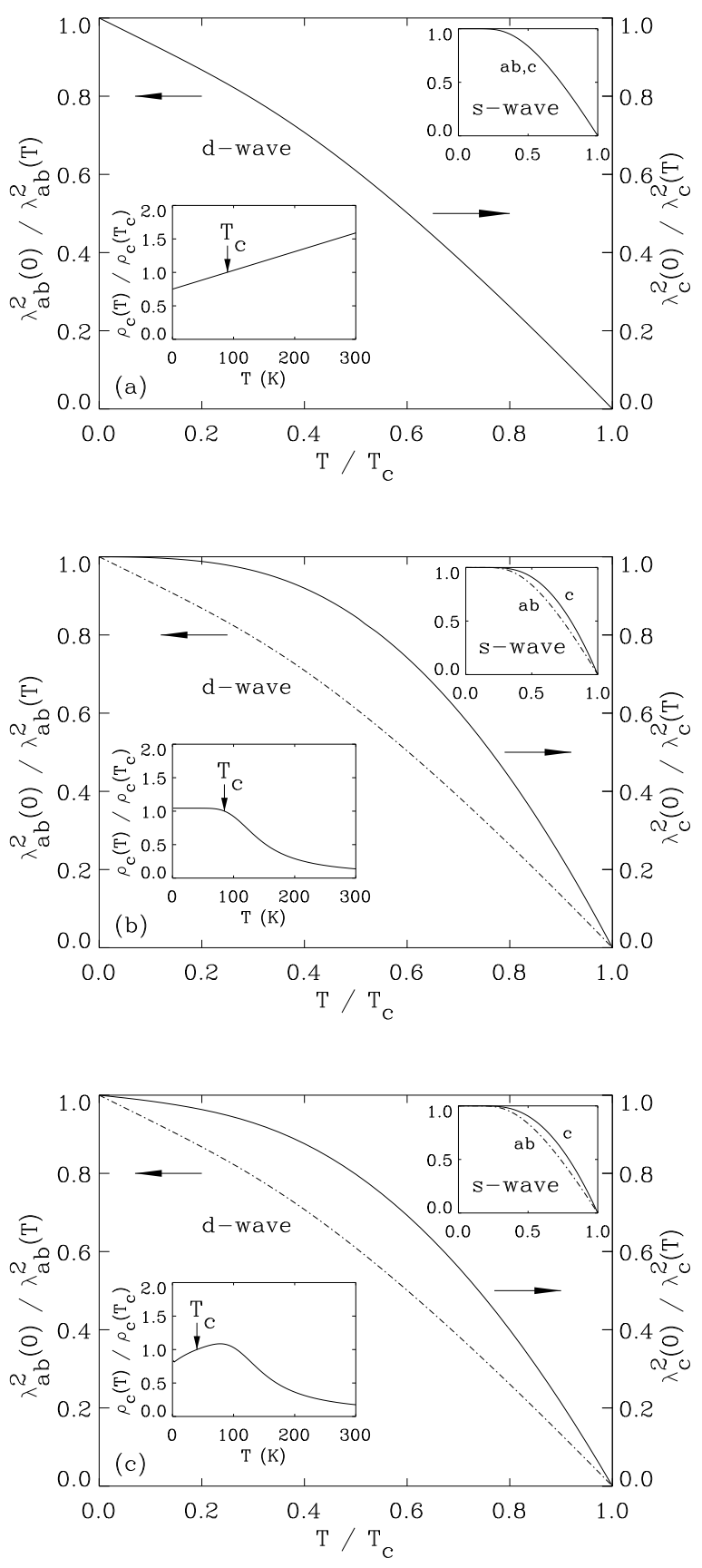

FIG. 1. Penetration depth ratios $\lambda_{a b}^{2}(0) / \lambda_{a b}^{2}(T)$ (solid line) and $\lambda_{c}^{2}(0) / \lambda_{c}^{2}(T)$ (dot-dashed line) as a function of the normalized temperature $T / T_{c}$ for both $d$-wave (main figure) and $s$-wave (upper right inset) pairing in different limits of the incoherent hopping model described in the text. The corresponding $c$-axis resistivity $\rho_{c}$ is shown in the lower-left inset normalized to its value at $T_{c}$ as a function of temperature. The limits illustrated are (a) direct hopping, which may be relevant for $\mathrm{YBa}_{2} \mathrm{Cu}_{3} \mathrm{O}_{6.9}$, (b) assisted hopping, which may be the case in $\mathrm{Bi}_{2} \mathrm{Sr}_{2} \mathrm{CaCu}_{2} \mathrm{O}_{8}$, and (c) a combination of these processes, which may obtain in underdoped compounds like $\mathrm{YBa}_{2} \mathrm{Cu}_{3} \mathrm{O}_{6.4}$. Note in (a) that both the $a b$ - and $c$-axis penetration depth ratios are the same when only direct hopping is present. 